\title{
Voltage Control by Means of Smart Transformer in Medium Voltage Feeder with Distribution Generation
}

\author{
Xiang Gao \\ Giovanni De Carne \\ Marco Liserre \\ Chair of Power Electronics \\ Kiel University \\ Kiel, Germany \\ Email: xg@tf.uni-kiel.de
}

\author{
Costas Vournas \\ School of Electrical and Computer Engineering \\ National Technical University of Athens \\ Athens, Greece \\ Email: vournas@power.ece.ntua.gr
}

\begin{abstract}
The integration of uncontrolled distributed generation (DG), like photovoltaic and wind turbines, and the growing amount of connected loads in the medium voltage (MV) grids have worsened the voltage variability, and in extreme cases violating the voltage limits. New mechanism and control action for supporting the voltage must be adopted, due to the unpredictability of these resources. The power electronics-based smart transformer (ST) can provide ancillary services to the distribution grid without the installation of additional devices, and among them the voltage and reactive power control. This paper illustrates, through simulation in PSCAD, the ST capability in controlling the voltage profile within the safe operation limits.
\end{abstract}

Index Terms-Smart transformer, distribution voltage control, distribution generation, reactive power control, MV grid control.

\section{INTRODUCTION}

The renewable energy sources (RES) represent nowadays an important energy resource. Considering just the solar and wind power generation in Germany, they have supplied respectively the $6 \%$ and $12.3 \%$ of the total electricity generation in 2015 [1]. However the power production of these resources does not correspond to load power demand, due to their intermittent power nature, causing conditions of high load/low generation and low load/high generation.

Due to the high $R / X$ ratio of lines in the MV grid, active power can influence the voltage, as well as the reactive power [2]. Considering that the DG connected to the MV grid can be in the size of several MW, if the low load consumption/high DG generation condition applies (e.g. during the vacations or weekends), it leads to a power flow reversal in the feeder, and the voltage at the DG terminals can rise above the overvoltage limit. If, instead, the high load consumption/low DG generation condition exists (e.g cloudly working-day without wind), the voltage at the end of MV feeder can drop even below the under-voltage limit.

In order to reduce the voltage variations of the MV grid in presence of DG, there are different mechanisms and control actions that have been proposed and analyzed [3]-[6]. These methods can be classified into two categories: $i$ ) using of traditional technology, e.g. transformer with load tap changer (LTC), and ii) using of power electronics devices, e.g. SVC.

The LTC transformer in the HV/MV substation can support the voltage profile by adjusting the distribution voltage setpoint. The drawback of this method is the influence on the voltage profile in all the MV grid. In other feeders connected to the same transformer the lower or upper voltage limit can be met, without the LTC controller noticing it [4]. The power electronics devices, such as Static Voltage Compensator (SVC), can support the voltage by means of reactive power control [6].

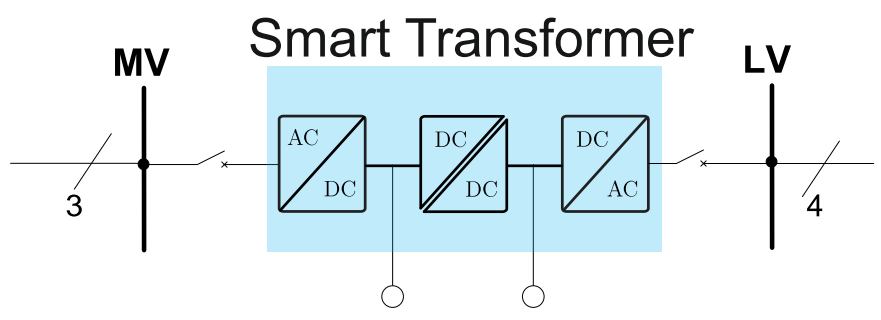

Fig. 1. ST in the grid

The power electronics-based smart transformer (ST) aims not only to adapt the voltage from the MV to the LV grids [7] (Fig. 1), but also to provide ancillary services. At MV side, the ST can perform grid current congestion [8], power factor correction and harmonics mitigation [9]. In comparison with SVC, ST in MV grid does not need to supply the reactive power of the load connected to the LV side of ST. Using ST to replace traditional MV/LV transformer, there is no need to install any other device to provide ancillary services.

The ST, providing reactive power in MV grid, can influence the voltage profile in the grid. The focus of this paper is on the performance of ST voltage support under different load consumption and DG power production conditions. 


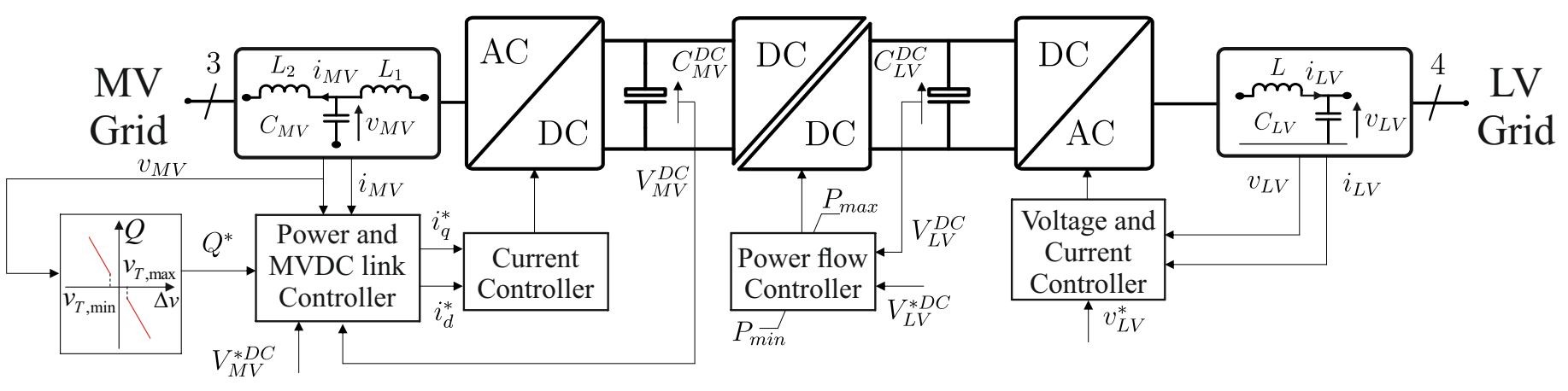

Fig. 2. The proposed structure and control scheme of ST

This paper is structured as follows: section II explains briefly the ST concept and control; section III introduces the MV grid investigated in this work; section IV presents the simulation results; eventually, the conclusions derived from simulation are given in Section V.

\section{SMART TRANSFORMER AND Voltage SUPPORT CONTROLLER}

\section{A. ST general configuration}

Although several topology solutions has been proposed [10], the basic control strategy is substantially the same for each ST solutions. Fig. 2 shows the basic control structure for a ST. The MV side controls the voltage in the MV DC link, regulating the active and reactive current absorption in the MV grid. Moreover, the reactive power is a degree of freedom for the ST MV converter: the ST can work unity power factor, or support the voltage providing reactive power. The reactive power setpoint can be set locally by a V/Q droop controller, or remotely from a centralized controller. This concept, together with the reactive power management algorithm, will be described more in detail in the next subsection.

The DC/DC converter has two main tasks: adapting the voltage from MV to $\mathrm{LV}$ and controlling the voltage level of the LV DC link. The DC/DC is regulated by means of a power flow controller and it regulates the power flow between the two DC stages to keep the LV DC link voltage at its nominal value. The power constraints are set between $P_{\max }$, determined by the ST sizing, and $P_{\min }$, that can be set or equal to $-P_{\max }$, or in order to avoid the reverse power flow, equal to zero.

The LV side controls the voltage waveform in the LV grid [7]. The ST provides a symmetrical voltage waveform with fixed amplitude and frequency independently from the load power request (e.g., high harmonic content). The current waveform is instead decided solely by the load.

In comparison with SVC, the ST can separate electrically the power flow between the MV and LV grids, by means of the 3-stage topology. A disturbance originated in the MV grid cannot propagate to LV grid via ST, and viceversa.

\section{B. ST controller of voltage support}

The ST can be interfaced in the distribution grid with different communication protocols and algorithms [7]. The reactive power control can be either central control that manages all the controllable resources in the MV grid, or local control using the measurements from the substation where ST is installed.

The reactive power control scheme proposed in this work is a local $V / Q$ droop controller shown in Fig. 2. The amount of reactive power injection is derived by means of a $V / Q$ droop characteristic curve, as depicted in Fig. 2. If the value of $\Delta v$ exceeds the deadband $\left(v_{T, \min }, v_{T, \max }\right)$, the droop controller is activated. The amount of reactive power is injected in proportion to the voltage variation. The droop controller is employed to sustain the voltage of the grid in case of fast voltage variations, e.g. voltage sags or swells.

The $Q^{*}$ is sent to the Power and MVDC link Controller to generate the new current reference $i_{q}^{*}$. The amount of reactive power that can be controlled is determined by the size of ST. The ST must respect always the active power demand of the LV grid. Thus the reactive power availability can be evaluated as (1):

$$
Q_{S T, \text { max }}= \pm \sqrt{S_{S T}^{2}-P_{\text {Load }}^{2}}
$$

\section{DESCRIPTION OF THE GRID UNDER TEST}

In order to analyze the ST voltage support service, one MV feeder of $11 \mathrm{kV}$ is modeled in PSCAD/EMTDC. The test MV feeder is derived from the UK distribution grid [11].

The main grid has a nominal voltage of $33 \mathrm{kV}$ with a short-circuit power of $600 \mathrm{MVA}$. The substation connects the main grid to the MV feeder through a $33 / 11 \mathrm{kV}, 15 \mathrm{MVA}$ transformer. The leakage impedance is $5 \%$. In this paper, it has been assumed that the transformer tap-changer is fixed to $1 \mathrm{pu}$. The grid under investigation is depicted in Fig. 3. The line impedance and the full load case are listed respectively in Table I and Table II.

The distributed generation is composed of a wind farm sized $9 \mathrm{MW}$ connected to Bus 25 and six photovoltaic power plants, each of $1 \mathrm{MW}$, connected to the grid as shown in Fig. 3. A unity power factor production has been assumed for all the distributed generation.

The simulation is divided into 2 parts. In the first part, steady-state for the extreme cases of high load consumption with low DG power generation, and low load consumption with high DG power generation are investigated. To show the 


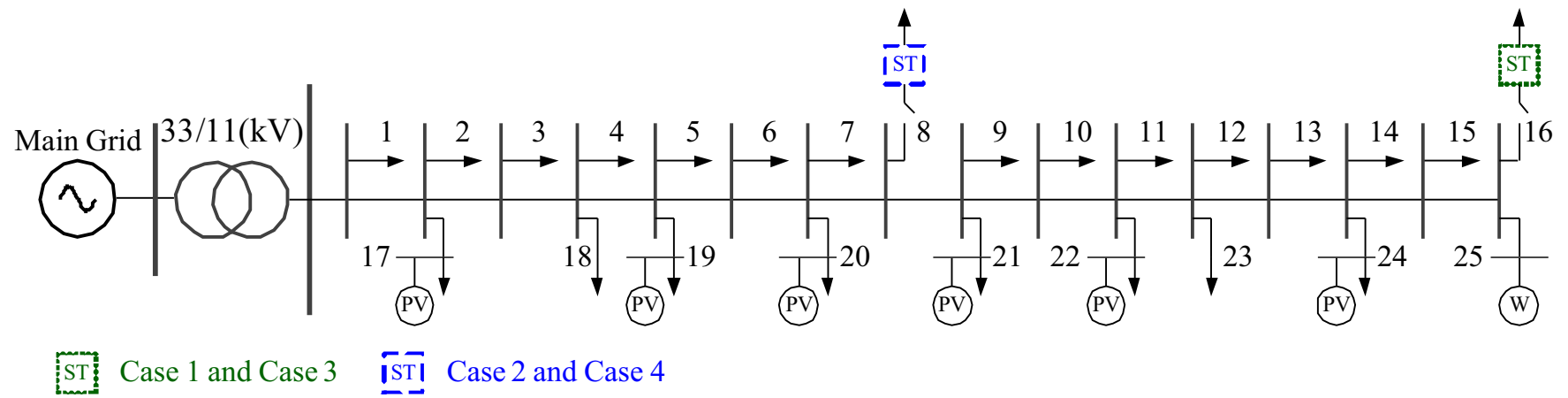

Fig. 3. Testing feeder

TABLE I

PARAMETERS OF POWER DELIVER LINES

\begin{tabular}{cccccccc}
\hline Bus & Bus & $\mathrm{R}(\Omega)$ & $\mathrm{X}(\Omega)$ & Bus & Bus & $\mathrm{R}(\Omega)$ & $\mathrm{X}(\Omega)$ \\
\hline 0 & 1 & 0.0665 & 0.0512 & 1 & 2 & 0.0665 & 0.0512 \\
2 & 3 & 0.0665 & 0.0512 & 3 & 4 & 0.0665 & 0.0512 \\
4 & 5 & 0.0665 & 0.0512 & 5 & 6 & 0.0665 & 0.0512 \\
6 & 7 & 0.0665 & 0.0512 & 7 & 8 & 0.0665 & 0.0512 \\
8 & 9 & 0.0665 & 0.0512 & 9 & 10 & 0.0665 & 0.0512 \\
10 & 11 & 0.0665 & 0.0512 & 11 & 12 & 0.0665 & 0.0512 \\
12 & 13 & 0.0665 & 0.0512 & 13 & 14 & 0.0665 & 0.0512 \\
14 & 15 & 0.0665 & 0.0512 & 15 & 16 & 0.0665 & 0.0512 \\
2 & 17 & 0.0729 & 0.0198 & 4 & 18 & 0.0729 & 0.0198 \\
5 & 19 & 0.0729 & 0.0198 & 7 & 20 & 0.0729 & 0.0198 \\
9 & 21 & 0.0729 & 0.0198 & 11 & 22 & 0.0729 & 0.0198 \\
12 & 23 & 0.0729 & 0.0198 & 14 & 24 & 0.0729 & 0.0198 \\
16 & 25 & 0.0729 & 0.0198 & & & & \\
\hline
\end{tabular}

TABLE II

LOAD PARAMETERS

\begin{tabular}{cccccc}
\hline Bus & P(MW) & Q(MVar) & Bus & P(MW) & Q(MVar) \\
\hline 1 & 0.57 & 0.144 & 2 & 0.57 & 0.144 \\
3 & 0.576 & 0.117 & 4 & 0.576 & 0.117 \\
5 & 0.576 & 0.117 & 6 & 0.576 & 0.117 \\
7 & 0.576 & 0.117 & 8 & 0.576 & 0.117 \\
9 & 0.576 & 0.117 & 10 & 0.576 & 0.117 \\
11 & 0.576 & 0.117 & 12 & 0.576 & 0.117 \\
13 & 0.576 & 0.117 & 14 & 0.576 & 0.117 \\
15 & 0.576 & 0.117 & 16 & 0.576 & 0.117 \\
17 & 0.372 & 0.0741 & 18 & 0.372 & 0.0741 \\
19 & 0.375 & 0.0747 & 20 & 0.375 & 0.0747 \\
21 & 0.375 & 0.0747 & 22 & 0.375 & 0.0747 \\
23 & 0.375 & 0.0747 & 24 & 0.375 & 0.0747 \\
\hline
\end{tabular}

performance of ST in different locations, 2 connection points, Bus 8 , in the middle of the feeder, and Bus 16 , at the end of the feeder, have been selected as installation point for the ST. The 4 test cases achieved combining the two grid conditions and two installation points are marked in Fig. 3.

Different scenarios are considered in each case study. The base case scenario $(S 0)$ represents the case where no ST is installed in the grid. In scenarios $1-3$, three different ST sizing have been considered. In scenario $1(S 1)$ the size of installed ST is 0.6 MVA, and in scenario 2 and $3(S 2$ and $S 3)$ the ST has been sized of $1 \mathrm{MVA}$ and 1.5 MVA respectively. The $Q / V$ droop controller gain assumed big enough that in every single simulation the reactive power output of ST is its maximal capability $Q_{S T, \max }$. The aims of this section are: $i$ ) to find out the influence of ST performance placed in different locations, and $i$ ) to investigate the minimum ST size for achieving good voltage control.

In the second part of simulation, the performances of ST in controlling the voltage are evaluated with 24-hour profiles of wind generation, solar generation and loads. The aforementioned profiles are shown in Fig. 5. In order to be consistent with the previous simulations, the ST is assumed in two different cases connected to Bus 8 and Bus 16 respectively. The size of ST is assumed to be identical.

The $Q / V$ droop controller gain has been adjusted to a constant $c_{d}$ based on (2).

$$
Q^{*}=c_{d} \cdot\left(U_{S T}-U_{r e f}\right)
$$

where $c_{d}$ is -20 , the unit is $(M V a r / p u) . U_{S T}-U_{r e f}$ is the voltage variation of bus where $\mathrm{ST}$ is connected to, and $U_{S T}$ and $U_{\text {ref }}$ are the measured voltage and its reference voltage in pu. If $U_{S T}>U_{r e f}$, the $\mathrm{ST}$ absorbs reactive power from MV grid. Viceversa, the ST injects reactive power in case of bus voltage lower than the reference one $\left(U_{S T}<U_{\text {ref }}\right)$. The characteristic of the $Q / V$ droop controller is shown in Fig. 4.

The control goal is to keep the voltage profile along the feeder within the safe limits [0.95 1.05] p.u.. The maximal voltage variation from the nominal value is used to analyze the performance of ST, as follows in (3).

$$
\Delta U_{\max }=\max \left|U_{i}-U_{\text {ref }}\right| \quad(i=1 \ldots 16)
$$

where $U_{\text {ref }}$ is $1 \mathrm{pu}$ in the simulation.

\section{Case Study}

In this section the steady-state analysis of extreme conditions (high load/low generation, low load/high generation) and the daily analysis have been performed. 


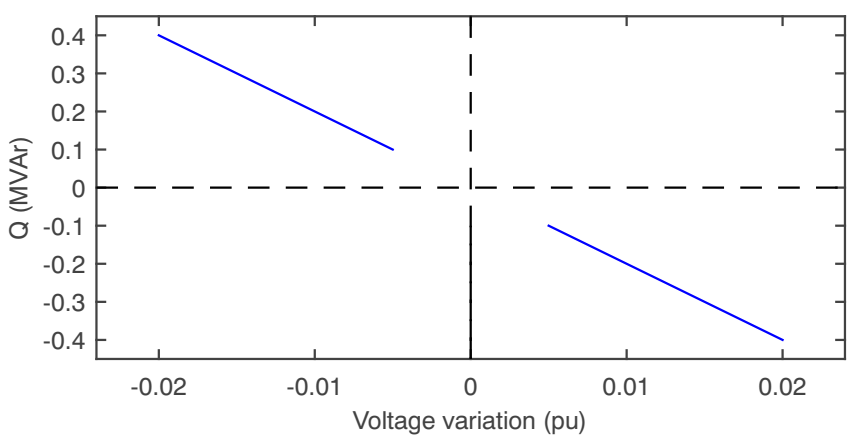

Fig. 4. Droop characteristic

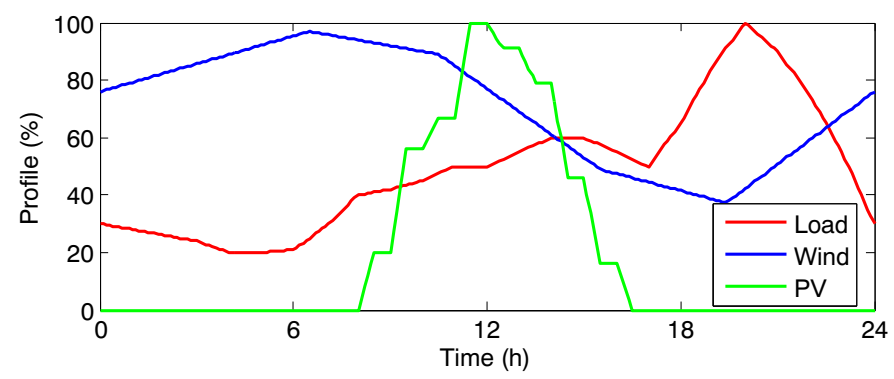

Fig. 5. Daily profiles

\section{A. Steady-state analysis}

In order to demonstrate the advantages of ST implementation, 4 case studies are simulated in PSCAD. Case 1 and Case 2 represent the high load consumption/low generation production condition, typical of the evening power consumption peak. The load absorbs the nominal power listed in Table II, instead the photovoltaic power plants are shut-down, and the wind power plant works at partial power, generating $3 \mathrm{MW}$. The two cases differ for the location of the ST: in Case 1, the ST is connected to Bus 16; in Case 2, the ST is connected to Bus 8. Case 3 and Case 4 are the cases describing the low load consumption/high power production, characteristic of the morning negative peak. The load is reduced to $25 \%$ of nominal load, and the DG is working at nominal power. Similarly to the first two cases, in Case 3, the ST is connected to Bus 16, and, in Case 4 to Bus 8 . The study cases are summarized in Table III.

TABLE III

DESCRIPTION OF CASES

\begin{tabular}{cccc}
\hline Case 1 & Case 2 & Case 3 & Case 4 \\
\hline $\mathrm{HC}^{1}$ & $\mathrm{HC}$ & $\mathrm{LC}^{3}$ & $\mathrm{LC}$ \\
$\mathrm{LG}^{2}$ & $\mathrm{LG}$ & $\mathrm{HG}^{4}$ & $\mathrm{HG}$ \\
$\mathrm{ST}$ at & $\mathrm{ST}$ at & $\mathrm{ST}$ at & $\mathrm{ST}$ at \\
Bus 16 & Bus 8 & Bus 16 & Bus 8 \\
\hline${ }^{1}$ High load consumption & \\
${ }^{2}$ Low DG power generation & \\
${ }^{3}$ Low load consumption \\
${ }^{4}$ High DG power generation
\end{tabular}

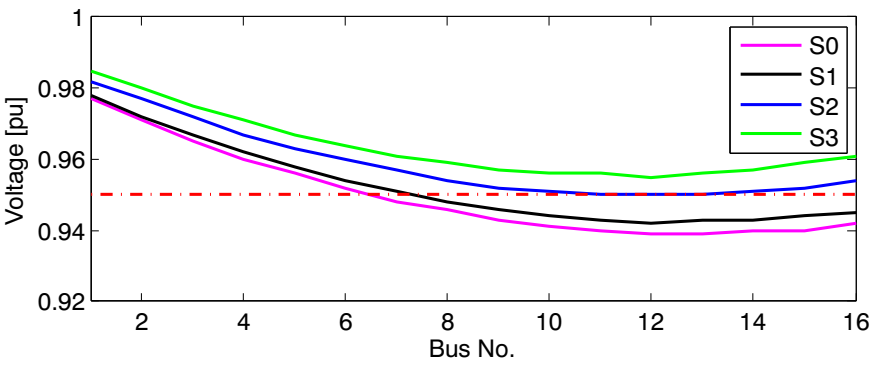

(a)

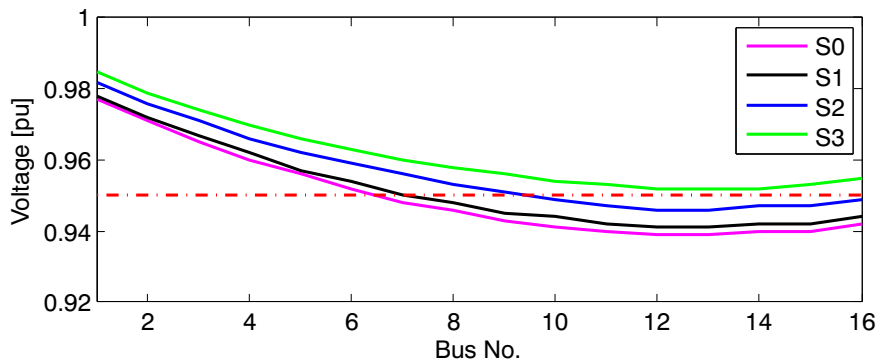

(b)

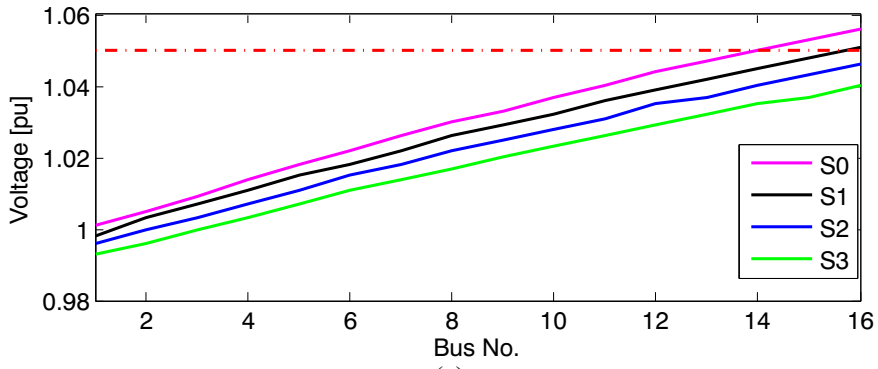

(c)

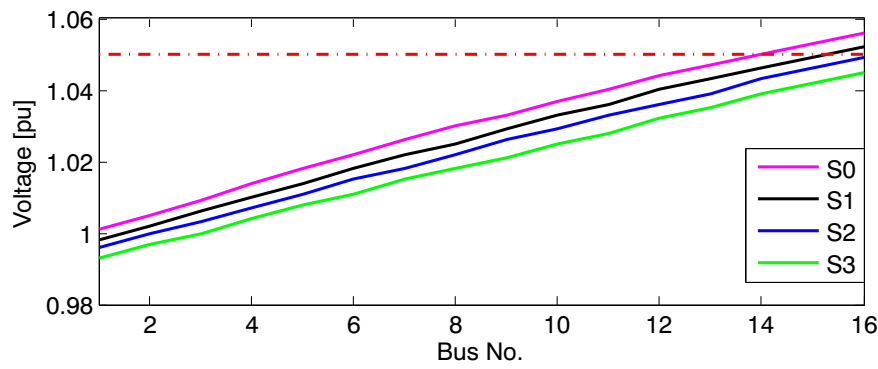

(d)

Fig. 6. Results of case study: (a) Case 1, (b) Case 2, (c) Case 3, (d) Case 4

The simulation results are presented in Fig. 6. In all four cases, the voltage tends to exceed the voltage limits of [0.95$1.05] \mathrm{pu}$, if no reactive power support is implemented (scenario $S 0$ in Fig. 6). In Case 1 and 2, the ST sustains the voltage profile, reducing the voltage drop along the feeder. However, a minimum ST size is required in order to avoid the lower voltage limit violation. In Case 1 , a ST size of $1 \mathrm{MVA}$ manages to sustain the voltage profile of the feeder within the constraints. If the ST is connected to Bus 8, only a size of 1.5 MVA achieves the voltage control in the safe limits [0.95$1.05] \mathrm{pu}$.

In case of high DG power production, like in Case 3 and Case 4, the ST reduces the voltage rise in the feeder. Similarly to Case 1 and Case 2, the ST is more effective if place at the 
end of the feeder. The control is achieved in both cases if the ST is sized for $1 \mathrm{MVA}$. However a sizing of 0.6 MVA is never fully able to control the voltage, independently from the position in the feeder. A size of 1.5 MVA achieves the full control of the feeder voltage profile.

The maximal voltage variations for different scenarios are computed using equation (3) and the results are summarized in Table IV.

TABLE IV

MaXimal Voltage Variation (P.U.)

\begin{tabular}{ccccc}
\hline & Case 1 & Case 2 & Case 3 & Case 4 \\
\hline S0 & 0.061 & 0.061 & 0.056 & 0.056 \\
S1(0.6MVA) & 0.058 & 0.059 & 0.051 & 0.052 \\
S2(1MVA) & 0.05 & 0.054 & 0.046 & 0.049 \\
S3(1.5MVA) & 0.045 & 0.048 & 0.04 & 0.045 \\
\hline
\end{tabular}

\section{B. Daily simulation case}

Considering the load and generation profile in Fig. 5, the voltage variations of Bus 13 to Bus 16 (furthest buses from the substation) are presented in Fig. 7. The size of ST both at Bus 8 and at Bus 16, is 1.5 MVA, with the capacity to fully control the voltage profile.

In Fig. 7(a), the case where no ST is implemented in the grid is simulated. It can be noted that an over-voltage occurs at Bus 16 in the morning before $7 \mathrm{am}$. It is caused by low load about $40 \%$ of its nominal value, and high wind generation production around $70 \%$ of its maximal power output. The photovoltaic is not producing at that time. Under-voltage conditions occur to several buses in the evening, where the load reaches its maximal amount and the generation has a limited output (wind generation around $60 \%$ of the nominal power and no photovoltaic at evening).

Analyzing Fig. 7(b) and Fig. 7(c), the ST is able to achieve the voltage control in both chosen placement, Bus 8 and Bus 16. Trivially the placement in Bus 16 obtains better results than the implementation in Bus 8. Especially for the over-voltage situation.

The reactive power injection of ST are shown in Fig. 8. It is observed that, during an over-voltage situation, the ST at Bus 16 injects reactive power into grid twice as the ST bus 8; during under-voltage situation, reactive power injection of the ST at Bus 16 is close to injection of ST at Bus 8 .

\section{CONCLUSION}

The increasing load demand along with the penetration of power from uncontrolled distributed generation (DG) in the distribution grids, like photovoltaic and wind turbines, has increased the voltage variability in medium voltage (MV) grids. In this paper, the service of ST voltage support in MV grid with DG has been studied. The performance of ST to reduce the voltage variation in the grid is simulated in a 25-bus MV feeder modeled in PSCAD/EMTDC. Cases of different load and DG power generation conditions, in different grid installation places of ST, and different size of

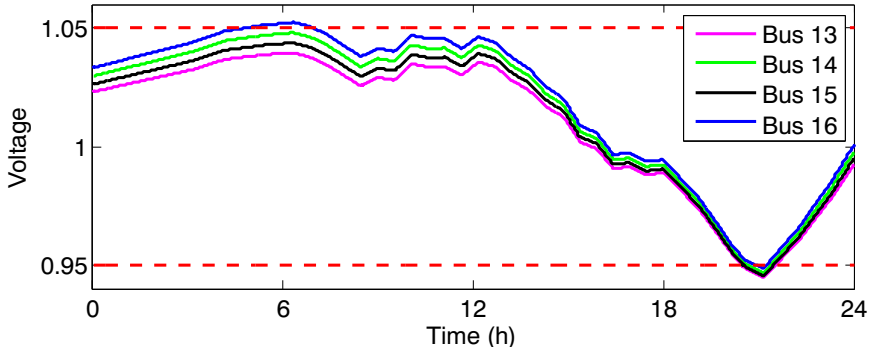

(a)

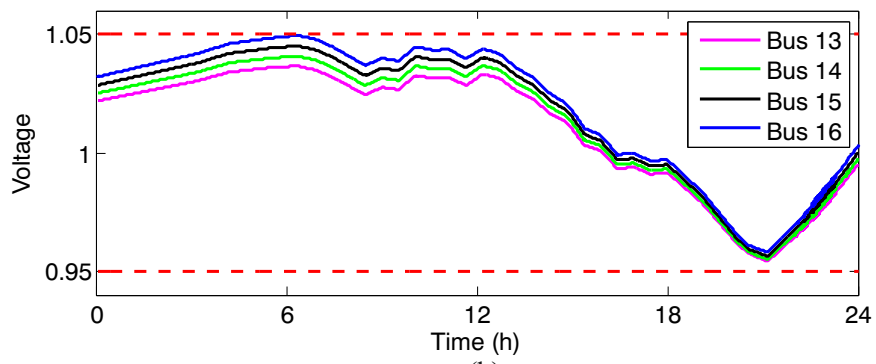

(b)

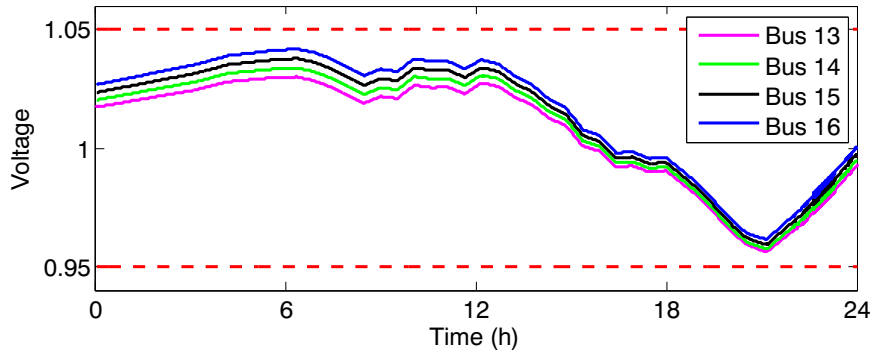

(c)

Fig. 7. Voltage variations from simulation of general grid conditions (a) No ST, (b) ST at Bus 8, (c) ST at Bus 16

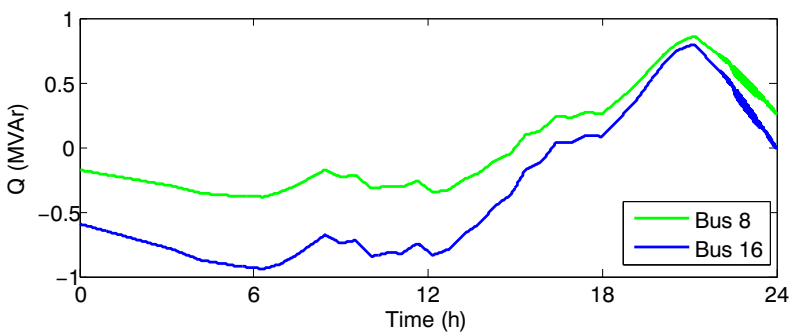

Fig. 8. Reactive power injection of ST in general grid conditions

ST are considered. The voltage profiles for all case studies without and with different sizes of ST are obtained by means of simulation. The paper illustrates, through simulation in PSCAD, the ST capability in controlling the voltage profile within the safe operation limits, without the installation of additional devices in the grid.

\section{ACKNOWLEDGMENT}

The research leading to these results has received funding from the European Research Council under the European Union's Seventh Framework Program (FP/2007-2013) / ERC Grant Agreement n. [616344] - HEART. 


\section{REFERENCES}

[1] Erneuerbare energien auf einen blick. Federal Ministry for Economic Affairs and Energy, Germany.

[2] J. von Appen, M. Braun, T. Stetz, K. Diwold, and D. Geibel, "Time in the sun: The challenge of high pv penetration in the german electric grid," IEEE Power and Energy Magazine, vol. 11, no. 2, pp. 55-64, March 2013

[3] C. Gao and M. A. Redfern, "A review of voltage control techniques of networks with distributed generations using on-load tap changer transformers," in Universities Power Engineering Conference (UPEC), 2010 45th International, Aug 2010, pp. 1-6.

[4] P. Jahangiri and D. Aliprantis, "Distributed volt/var control by pv inverters," in 2014 IEEE PES General Meeting - Conference Exposition, July 2014, pp. 1-1.

[5] Y. Agalgaonkar, B. C. Pal, and R. A. Jabr, "Distribution voltage control considering the impact of pv generation on tap changers and autonomous regulators," in 2014 IEEE PES General Meeting - Conference Exposition, July 2014, pp. 1-1.

[6] P. M. S. Carvalho, P. F. Correia, and L. A. F. M. Ferreira, "Distributed reactive power generation control for voltage rise mitigation in distribution networks," IEEE Transactions on Power Systems, vol. 23, no. 2, pp. 766-772, May 2008.

[7] M. Liserre, G. Buticchi, M. Andresen, G. De Carne, L. Costa, and Z.$X$. Zou, "The smart transformer, its impact on the electric grid and its technology challenges," IEEE Trans. Ind. Electr. Mag., in press 2016.

[8] G. D. Carne, M. Liserre, K. Christakou, and M. Paolone, "Integrated voltage control and line congestion management in active distribution networks by means of smart transformers," in 2014 IEEE 23rd International Symposium on Industrial Electronics (ISIE), June 2014, pp. 2613-2619.

[9] C. Kumar and M. Liserre, "Operation and control of smart transformer for improving performance of medium voltage power distribution system," in 2015 IEEE 6th International Symposium on Power Electronics for Distributed Generation Systems (PEDG), June 2015, pp. 1-6.

[10] X. She, A. Q. Huang, and R. Burgos, "Review of solid-state transformer technologies and their application in power distribution systems," IEEE Journal of Emerging and Selected Topics in Power Electronics, vol. 1, no. 3, pp. 186-198, Sept 2013.

[11] United kingdom generic distribution network (ukgds). [Online]. Available: http://sedg.ac.uk. 